\title{
Rechtsgeschichte
}

http://www.rg-rechtsgeschichte.de/rg5

$\operatorname{Rg} 52004 \quad 49-54$

Zitiervorschlag: Rechtsgeschichte Rg 5 (2004)

http://dx.doi.org/10.12946/rg05/049-054

\section{Michael Stolleis}

Wer Solidarität sagt, will etwas haben 


\section{Abstract}

»Solidarity« can only have the meaning social communication attributes to it over time. The word occurs in the 19th century when former structures are conceived as being in crisis. The invocation of solidarity was meant to call upon a feeling of unity and support against internal and external threats. The workers' movement, the Church's social doctrines, social reforms, civil and constitutional theory (including their authoritarian offshoots), all refer to the notion of solidarity. Using the term was always meant in an educational, a demanding, a normative way. Most of the concrete demands based upon this concept are nowadays basic elements of national and European constitutional and social law. Thus the question whether "solidarity" should serve as the necessary moral completion of formal law remains unanswered. But the fact that the word today no longer finds a common consensus indicates that its zenith is already passed. 


\section{Wer Solidarität sagt, will etwas haben}

»Solidarität « ist ein Wieselwort von gallertartiger Konsistenz. Es ist allgegenwärtig, gibt sich bedeutungsschwer und meist auch etwas vorwurfsvoll. Sein Kontext ist durchweg normativ. Es taucht dort auf, wo es um den Appell an Personen auf der gleichen Ebene geht. Typischerweise rufen Gruppen nach »Solidarität ", deren innere Bindungen bröckeln oder die sich in Gefahrenlagen zusammenscharen. Als es noch Standesgenossen gab, erinnerten sie gerne an die Solidarität, wenn es darum ging, den Zusammenhalt derselben Schicht zu wahren. Die alten Zünfte, Gilden, Gaffeln, Einungen und Genossenschaften waren Solidaritätsverbände. Die Arbeiterbewegung übernahm hiervon nicht nur das Wort »Genossen «, sondern auch den Appell an die Klassensolidarität. Und selbst wer heutzutage einen Krieg führen will, erinnert an alte Dankesschulden und appelliert an die Solidarität der Bundesgenossen.

Natürlich gibt es kein »Wesen « der Solidarität, keinen interpretierbaren festen »Begriff «. Was es gibt, sind die verschiedenen Redeweisen von Solidarität, die wechselnden Zusammenhänge innerhalb der Texte sowie vor allem die in den Sprechgemeinschaften jeweils evozierten Assoziationen. Was Solidarität historisch bedeutete oder heute bedeutet, lässt sich demnach nur durch spezifische Textanalyse feststellen. So zeigt sich im I9. Jahrhundert zunächst eine revolutionär sozialistische, später sozialdemokratische Linie der Gewerkschaften und der dazugehörigen politischen Partei. Ihnen ist "Solidarität « die Parole der Arbeiterklasse, die Menschen gleicher Klassenlage zusammenführen will, um sich gegen das »Kapital «, die »Arbeitgeber « oder den mit ihnen verbündeten Staat zu behaupten.
Gleichzeitig kommt aus der Bewegung der Associationen und Vereine, aus den Hülfscassen und Genossenschaften eine bürgerlich getönte »Solidarität «. Sie bezieht das Wort zunächst nur auf den unmittelbar verfolgten Zweck, der karitativer, sozialer oder sonstwie altruistischer Natur ist. Daneben schwingt bei »Solidarität « die Abwehrfront gegen den »Vierten Stand « mit. Bürgerliche Vereinsgründung dient den Vereinszwecken, aber in größerer Perspektive der Selbstorganisation der Gesellschaft, die stark sein soll, stark gegen den Obrigkeitsstaat, der diese Selbstorganisation misstrauisch beäugt und polizeilich kontrolliert, stark aber auch als Bollwerk gegen die »entwurzelten « Massen der Arbeiterbewegung. In diesem Sinn haben Robert von Mohl, Rudolf von Gneist, Otto Bähr, Lorenz von Stein und vor allem dann Otto von Gierke die solidarisch organisierte Gesellschaft forciert und deren historische Legitimation ausgebaut, um dem Bürgertum eine Plattform zu geben, auf der es in jenem Zweifrontenkrieg stehen könne.

In diese Richtung zielte auch die Enzyklika »Rerum novarum « ( I89I). Die katholische Soziallehre, normativ hoch besetzt, entwarf eine antiliberale, organisch von unten nach oben aufgebaute, solidarische Gesellschaft. Vor allem die französische Sozialphilosophie des ausgehenden I9. Jahrhunderts nahm das Stichwort »Solidarität « auf und entwickelte daraus eine vermittelnde bürgerliche Sozialphilosophie. ${ }^{\mathrm{I}}$ In der kirchennahen Variante stand Christi Opfertod am Kreuz für Gottes »Solidarität « mit den Menschen, aber auch für das Gebot der Nächstenliebe unter Menschen sowie für ein immanent an den kirchlichen Geboten orientiertes wirtschaftliches Verhalten, das den Egoismus altruistisch zügeln sollte. ${ }^{2}$

\footnotetext{
I J.E. S. HaYward, Solidarity: The Social History of an Idea in I9th Century France, in: International Review of Social History 4 (I959) 26I. Das Hauptwerk der Richtung stammt von L. BouRGEOIS, La Solidarité, I 897 u. ö.

2 H. Pesch, Lehrbuch der Nationalökonomie, I, I905, 2. Aufl. Freiburg I9I4. Siehe ANTON Rauscher, Solidarität, in: Staatslexikon der Görres-Gesellschaft,
}

7. Aufl. Freiburg I988, Bd. 4, II9I-II94. 
Bürgerliche und antibürgerliche Hoffnungen auf einen »Dritten Weg « zwischen Kapitalismus und Kommunismus führten im Fin de Siècle zu allerlei Experimenten, Kolonien und Landkommunen, bündischer Bewegung, Jugendbewegung und Männerbünden. ${ }^{3}$ So bunt dies alles sein mochte, die durchgängigen Tendenzen richteten sich auf Abbau von Hierarchien, also auf Gleichheit, auf Appelle an den Altruismus, Überwindung von Spannungen durch Heranziehung eines neuen Menschen, der willig und fähig war, "Solidarität « zu üben. Die autoritäre Variante dieser Sehnsüchte enthielt den immer lauter werdenden Ruf nach Führertum und Führer, zugleich mit der Unterwerfungsbereitschaft unter den Willen eines Einzelnen, besiegelt durch den Treueschwur, der Gemeinschaft treu zu bleiben »bis in den Tod «. Anarchistische und autoritäre Ansätze nötigten auf diese Weise gleichermaßen, fast wider Willen, zu solidarischer Gruppenbildung und zur Werbung mit den Zauberworten »Gemeinschaft « und »Solidarität «. Dass sozialistische, bürgerliche und präfaschistische Bewegungen der zwanziger Jahre voller Gemeinschaftsrhetorik waren, ist ebenso bekannt ${ }^{4}$ wie deren spätere Umsetzung in rechtliche Kontexte. Solidarietà war ein Kernbegriff des faschistischen Arbeitsrechts, während der Nationalsozialismus die sozialistisch kontaminierte Solidarität vermied und es vorzog, von der die Klassengegensätze überwindenden Volksgemeinschaft zu sprechen. Die auf Schicksalsgenossen bezogene horizontale Solidarität verschwand damit in der Solidarität aller mit allen, die inhaltlich entsprechend entleert war. Damit schob sich die vertikale Befehlsstruktur des Führertums nach vorne.

Geht man in der Rechtsgeschichte noch einmal zwei Generationen zurück in die Zeit der Reichsgründung nach I87 I, dann erkennt man dort die Anfänge einer vielfältig mit Solidaritätsrhetorik drapierten Gedanken- und Gesetzgebungswelt. Es ist die Zeit der sozialistischen und der christlichen Gewerkschaftsbewegung, der Entstehung der auf Solidarität gegründeten Verbände der Industrie und der Landwirte, der landwirtschaftlichen Genossenschaften ${ }^{5}$ und des Genossenschaftsgesetzes (I 889). Alle diese Aktivitäten nutzten die rechtlich und faktisch garantierte Assoziationsfreiheit. Ihre Grundidee lag in der Beobachtung, dass organisatorisch gebündelte Interessen den individualisierenden Kräften des Markts besser gewachsen waren. Wer sich »solidarisch " vereinigte, hatte sowohl als Käufer wie auch als Verkäufer eine größere Chance, die Konditionen zu bestimmen. Unter der Parole "Alle für einen, einer für alle« war der Appell an die Solidarität ein Erfolg versprechendes Programm in einer Zeit, die gerade den Pauperismus und die Phase des Hochliberalismus erfahren hatte und im Mittelstand (Landwirtschaft, Handwerk) den Wegfall der früher schützenden intermediären Institutionen schmerzlich spürte. Selbsthilfe durch die Solidarität der wirtschaftlich Gleichgestellten erschien deshalb als zentrales gesellschaftspolitisches Programm zwischen staatlicher Bürokratie und dem gefährlich offenen freien Markt.

Vor allem aber im Umfeld der 1883 bis I 889 geschaffenen "Arbeiterversicherung « berief man sich auf Solidarität. Das ergab eine eigentümliche Mischung von christlichem Paternalismus, Staatssozialismus und Selbsthilfe, die in wechselnden Anteilen die Kranken-, Unfall-, Altersund Invaliditätsversicherung prägten. ${ }^{6}$ In der Krankenversicherung (I 883) sollten die Gesunden mit ihren Beiträgen solidarisch für die Kranken einstehen, desgleichen die verdienenden »Ernährer« für die mitversicherten Familienangehörigen, im weiteren Verlauf dann die ver-

\footnotetext{
3 U. Linse, Barfüßige Propheten. Erlöser der zwanziger Jahre, Berlin I983; DERs. Die Jugendbewegung, in: Das wilhelminische Bildungsbürgertum, hg. von K. VondunG, Göttingen I976, I I9-I37; Zurück, o Mensch, zur Mutter Erde. Landkommunen in Deutschland I 890-I933, hg. von U. LINSE, München I983. Reiche Nachweise auch in: Männerbande, Männerbünde, Katalog zur Aus-
} ั. stellung, hg. von G. VöLGER und K. v. WELCK, Köln I990, darin insbes. J. Reulecke, Das Jahr I 902 und die Ursprünge der Männerbund-Ideologie in Deutschland, Bd. I, 3 ff.

4 M. Stolleis, Gemeinschaft und Volksgemeinschaft, in: Vierteljahrshefte für Zeitgeschichte 20 (I972) I 6-38.

5 Etwa der Darlehenskassenvereine von Fr. W. Raiffeisen (I 8 I 8-I 888), die auf dem Solidarprinzip zugunsten des bäuerlichen Mittelstands beruhten. Heute bilden die Raiffeisenbanken ein dichtes Netz genossenschaftlich organisierter Institute in Österreich, Deutschland und der Schweiz.

6 M. Stolleis, Geschichte des Sozialrechts in Deutschland, Stuttgart $2003,36 \mathrm{ff}$. 
dienenden Versicherten für die nicht verdienenden Versicherten. In der Unfallversicherung (I 884) zahlen die Betriebe »solidarisch" eine Umlage, um das Risiko Betriebsunfall unter Verzicht auf die individuelle Schuldfrage zu neutralisieren. Die branchenverwandten Betriebe wurden zu »Berufsgenossenschaften « zusammengeschlossen. Die Rentenversicherung (I889) sparte zunächst nur die Beitragssummen treuhänderisch an, um sie dann, wenn der Versicherungsfall eintrat, auszuzahlen. Aber auch hier waltete »Solidarität «: Alle opferten Beiträge für die wenigen, die eine Rente erwarten durften. Nachdem die Witwen- und Waisenrente durchgesetzt war, erweiterte sich die eheliche und elterliche Solidarität gewissermaßen über den Tod hinaus. Seit der Umstellung der Alterssicherung auf das Umlageprinzip und den Generationenvertrag (I957) zahlten die Jungen für die Alten, und wenn dies nicht reichte, sprang der Staat solidarisch mit einem Staatszuschuss ein (Art. I20 GG). Inzwischen sind Handwerker und Landwirte, Künstler und andere Freiberufler in diese große »Solidargemeinschaft « aufgenommen worden. Wer dort nicht Platz fand, organisierte sich in »Berufsständischen Versorgungswerken «, die ebenfalls vom Grundgedanken der Solidarität getragen werden. Gleiches ließe sich von der Arbeitslosenversicherung und der Pflegefallversicherung sagen.

Seit jedoch vor drei Jahrzehnten die ersten Maßnahmen zur Kürzung der Sozialleistungen erlassen wurden - es handelte sich um das euphemistisch so genannte Haushaltsstrukturgesetz $1975^{7}$-, riss die Kette der Einschnitte in die vorher viel zu breit angelegten Leistungskataloge nicht mehr ab. Je tiefer diese von der Finanzlage diktierten Einschnitte waren, desto stärker trat die Rhetorik der Solidarität in die Lücke. Sie sollte auspolstern, was den Systemen an Ein- schnitten zugemutet wurde. Nun war von der Solidarität der Generationen ( Generationenvertrag«), der Geschlechter, der Altersstufen, der Arbeitsbesitzer und der Arbeitslosen, der Gesunden mit den Kranken, der Träger geringer mit den Trägern hoher Risiken, kurz: aller mit allen die Rede.

Aber diese Rede galt einem Phantom. In der Lebenswirklichkeit kam Solidarität als verlässliche Kraft nicht mehr vor. Die ursprünglich zur Repräsentation der Versicherten geschaffene Selbstverwaltung ist längst zu unförmigen Bürokratien versteinert. Von einer die Betroffenen emotional erreichenden "Solidarität « kann bei einer modernen, EDV-gesteuerten Volksversicherung, die neun Zehntel der Bevölkerung umfasst, keine Rede sein. Das allseits versicherte Publikum weiß kaum, dass es "Sozialwahlen « gibt die Wahlbeteiligung ist extrem gering. So genannte Friedenswahlen, bei denen auch die Wahlmöglichkeiten beseitigt sind, ersticken den letzten Rest an partizipatorischer Auseinandersetzung. Die Sozialversicherung ist der bedeutendste innenpolitische Gegenstand der parlamentarischen Gesetzgebung. Ihre Steuerung liegt völlig in den Händen der Ministerien, der Verbände und der Parteien. Diese verbinden ihr Schicksal mit dem Überleben des »bewährten Systems «, obwohl es inzwischen zum Talkshow-Wissen gehört, dass die rasche Alterung der Gesellschaft wegen steigender Lebenserwartung auf der einen und gesunkener Kinderzahlen auf der anderen Seite das bewährte System in der nächsten Generation zum Stillstand bringen wird.

Mit der Berufung auf »Solidarität « ist jedenfalls dem Zwang zu einer durchgreifenden Verschlankung und prinzipiellen Neukonstruktion des Systems der sozialen Sicherung nicht mehr beizukommen. "Solidarität « ist ein Ruf aus dem

\footnotetext{
7 Haushaltsstrukturgesetz vom I 8. Dezember I975, BGBl I, $309 \mathrm{I}$.
} 
I9. Jahrhundert. Schon damals war er ein ideologisches Versatzstück des Klassenkampfs und der dabei aufgebauten bürgerlichen Abwehrfronten. Man sollte auf den Terminus heute verzichten und der Tatsache ins Auge blicken, dass die industrielle Massengesellschaft die »ständischen « Strukturen, innerhalb derer Solidarität möglich zu sein scheint, weder benötigt noch haben will. Deshalb ist das unklare Sprechen von Solidarität eher verwirrend. Schaut man durch die ideologische Nebelwand, dann zeigt sich: Wer Solidarität sagt, will etwas haben. Zur Umverteilung reichen jedoch Appelle nicht hin. Es bedarf parlamentarischer Mehrheiten, und es bedarf der Implementation in ein von Besitzständen und Besitzstandsgarantien starrendes System.

Das Grundgesetz fordert in sehr allgemeinen Worten, der Staat solle Sozialstaat sein, den Gleichheitssatz und die als Eigentum gedeuteten Anwartschaften auf Leistungen beachten. Diese Ansatzpunkte sind in fünfzig Jahren Rechtsprechung und Gesetzgebung solide und in allen Feinheiten ausziseliert worden. ${ }^{8}$ Jede Änderung muss sich an den genannten Postulaten messen lassen, nicht jedoch daran, ob die Änderung dem Prinzip der "Solidarität « entspricht. Die Richter wären auch überfordert, wenn sie diesen Maßstab anwenden sollten. Auf einen für die Mehrheit gültigen festen Lebenszusammenhang oder tradierte Ansichten, was Solidarität bedeuten soll, könnten sie nicht zurückgreifen. Die Gesetzestexte und deren Kommentare, die Solidarität nicht verwenden, ließen sie im Stich. Die Lexika, die man heranziehen könnte, geben überwiegend historische Nachrichten darüber, was unter Solidarität verstanden wurde. Der Rückgriff auf das Alltagswissen, die herkömmliche Zuflucht für den unter Entscheidungszwang stehenden Richter, wenn er nicht mehr weiter weiß, versagt hier ebenfalls, und es ist leicht zu verstehen, warum. Die Menschen agieren, wenn man den soziologischen Befunden glauben darf, kaum noch »solidarisch « in fest gefügten Gruppen, denen man lebenslang zugehört. Für die westlichen Industriegesellschaften ist eher typisch, dass die Biographien und Karriereverläufe ihr Gleichmaß verloren haben. Das Individuum setzt häufiger als früher mehrfach an, wechselt Plätze und Rollen und sichert sich weniger in starren Hierarchien ab als in mehrfach geknüpften Netzwerken. Viele Rollen werden gleichzeitig wahrgenommen, eine Festlegung auf eine »Identität « wird schwieriger. Die meisten sind Arbeitnehmer, Konsumenten, Bürger, Wähler, Schutzbedürftige und Risikoträger in einer Person. Das führt zu politischen Paradoxien, in denen sich auch die traditionelle Unterscheidung von »rechts « und »links « aufzulösen scheint; denn der Einzelne kann sowohl Steuersenkung fordern als auch gegen die Reduzierungen von staatlichen Leistungen protestieren, kann als Unternehmer Risikobereitschaft praktizieren, aber als Kranker jedes Risiko scheuen, kann Solidarität auf der einen Seite predigen und auf der anderen Abschreibungsmöglichkeiten exzessiv nutzen oder gar Steuerbetrug begehen. Die gesellschaftlichen Leitbilder changieren deshalb auch. Talkshows, politische öffentliche Rede und Werbung favorisieren den durchsetzungsfähigen, seinen Vorteil nutzenden homo oeconomicus, Spendenaufrufe zur Linderung von Armut, Naturkatastrophen oder mangelhafter Ausbildung appellieren an »Solidarität «. Staat und Privatversicherungen fordern auf, die Absicherung vor Lebensrisiken in Solidarsystemen zu suchen. Der homo oeconomicus spendet und sorgt vor, und er kann dies so begründen, dass wirklich kluges ökonomisches Denken die Solidarabgaben als langfristig nützlich in die

8 D. GRIMM, Solidaritätsprinzip,

in: Evangelisches Staatslexikon,

3. Aufl. Stuttgart I 987 f., Bd. 2, $3 \mathrm{I} 46 \mathrm{f}$. 
Rechnung einbringen kann. Uneigennützige Solidarität verliert so ihr Terrain; denn alles muss sich irgendwie » rechnen «.

Trifft dies alles nur halbwegs zu, dann ist doch erstaunlich, wie viel Zutrauen sich in der heutigen Rechts- und Sozialphilosophie findet, "Solidarität « begrifflich zu fassen und operationalisierbar zu machen. Liberalismuskritik aus der Perspektive der katholischen Soziallehre, kommunitaristische Ergänzungen oder Verwandlungen des amerikanischen Liberalismus, sozialdemokratische Vorbehalte gegen die "gleiche Ungleichheit « der Marktgesellschaft, aber auch konservative Verantwortungsethik gegenüber dem Schwächeren treffen hier zusammen. Die normativen Sätze der unter dem plakativen Wort »Solidarität « segelnden Ethik haben ihre historischen Wurzeln, wie neuerdings Hauke Brunkhorst beschrieben hat, ${ }^{9}$ in der republikanischen Bürgerfreundschaft der antiken Polis, in der christlichen universellen Nächstenliebe sowie in der aus Aufklärung, Gleichheits- und Gesetzespathos hervorgegangenen "Fraternité «. ${ }^{\text {Io }}$ So unbestreitbar dies historisch ist und so menschlich sympathisch oder gesellschaftspolitisch klug die Postulate sein mögen, so wenig gelingt es, sie als verbindliche Sätze zu etablieren. Solidarität bleibt ein Kondensat der jeweils privaten Wünsche, wie eine Gesellschaft auszusehen habe, nämlich freiheitlich und rücksichtsvoll, dynamisch, aber nicht selbstzerstörerisch, angetrieben durch das Recht auf Selbstentfaltung, aber gebremst durch die respektvolle Wahrnehmung der Rechte anderer. Es soll eine Gesellschaft sein, welche die Menschenwürde materiell und ideell garantiert, kurzum eine plurale demokratische Zivilgesellschaft.

Erhard Denninger etwa hat vor einer Generation in einer subtilen Auseinandersetzung mit Max Schelers »Solidarismus« dessen Kon- zept reziproker Verantwortungsbeziehungen als Grundlage einer liberalen Staatstheorie genutzt. ${ }^{\text {II }}$ Ihre Basissätze waren und sind unverändert die Anerkennung der »relativen Subjektivität aller Werterfahrung", der "Verzicht darauf, den anderen zur Glückseligkeit zwingen $\mathrm{zu}$ wollen ${ }^{\mathbf{I}}{ }^{\mathbf{2}}$ der Respekt vor der anderen Person, die Vermutung für die Freiheit (in dubio pro libertate), die einheitliche sozialethische Fundierung von Rechts- und Sozialstaatsprinzip und die Konsensorientierung des offenen politischen Prozesses. Denninger möchte deshalb heute - das ist seine konkrete Utopie - die klassische Trias von »Liberté, Egalité, Fraternité« durch »Vielfalt, Sicherheit, Solidarität « ergänzen. ${ }^{\text {I3 }}$ Von diesen dreien ist jedenfalls Solidarität, ungeachtet ihrer gelegentlichen Positivierung im Sozialrecht, ein eindeutig moralisches Prinzip. ${ }^{\text {I4 }}$ Als solches wird es gebraucht, und zwar zur Stützung des Formalrechts, denn dieses empfange »belebende Kraft und innere Rechtfertigung aus seiner Verbindung mit moralischen Prinzipien ..., wie umstritten und zerbrechlich diese auch sein mögen «. ${ }^{5}$ Denninger nah verwandt sind die Überlegungen von Günter Frankenberg, der in der gegenwärtigen Gesellschaft einen »krassen Mangel an Solidarität « konstatiert, also an Mitgefühl mit denen, die es brauchen,

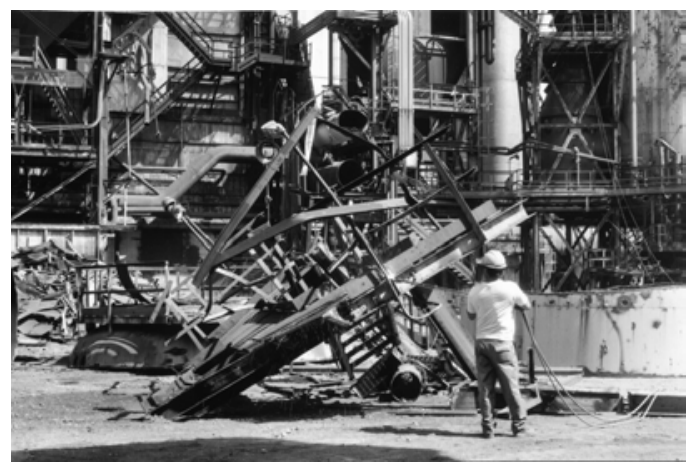

9 H. BRUNKHORST, Solidarität. Von der Bürgerfreundschaft zur globalen Rechtsgenossenschaft, Frankfurt 2002.

IO W. SCHIEdER, Brüderlichkeit, in: O. Brunner, W. Conze, R. KoSELLECK, Geschichtliche Grundbegriffe, Bd. I, Stuttgart 1972 (Nachdr. I979) 552-58I. - Eine kurze Skizze zur Abgrenzung von Brüderlichkeit und Solidarität bei H. Hofmann, Einführung in die
Rechts- und Staatsphilosophie, Darmstadt 2000, I93.

i I E. Denninger, Rechtsperson und Solidarität, Frankfurt, Berlin 1967.

I 2 Denninger (Fn. II) 2 I 8.

I3 E. Denninger, Menschenrechte und Grundgesetz, Weinheim I994, 6I.

I4 Dass sie "von Haus aus « ein Rechtsbegriff war und, wie Hasso Hofmann mit Recht feststellt, die »kollektive genossenschaftliche
Verantwortung für die Schuld eines Einzelnen bezeichnet" (HoFMANN, Einführung [Fn. Io] I93), ändert daran nichts. Entscheidend für das heutige Verständnis sind die jetzt mitschwingenden, etwa seit der Mitte des I9. Jahrhunderts bekannten Kontexte der fraternité, der ethischen Verantwortung für Andere, insbesondere Schwächere oder Ungeborene.

I 5 Denninger (Fn i i) 9. 
um als Freie und Gleiche mit anderen leben zu können. ${ }^{16} \mathrm{Da}$ er annimmt, Solidarität habe » auf gesamtgesellschaftlicher Ebene keine Chance «, ${ }^{\mathbf{I 7}}$ konzentriert er sich auf den Sozialstaat und hält dort Grundsicherung, Hilfe zur Selbsthilfe und generell einen »sozial gemilderten « Liberalismus für Ausprägungen des Solidaritätsprinzips. Dieses wiederum ist Voraussetzung für die Zivilgesellschaft, in der demokratische Partizipation in öffentlicher Auseinandersetzung unter Gleichen nur möglich ist, wenn ein relatives materielles Gleichheitsniveau erreicht ist.

Gewiss können die Theoretiker der Solidarität versuchen, diese Postulate aus höheren Prinzipien abzuleiten (I) oder anhand von Utilitätsüberlegungen plausibel machen, dass solidarisches Verhalten kurz- und langfristig größeren Nutzen bringt (2), sie können sich auf intuitive Werterfahrung berufen (3) oder darauf vertrauen, im herrschaftsfreien Diskurs werde sich ein auf Solidarität beruhendes Gesellschaftsmodell herauskristallisieren (4). Auf jedem dieser vier Wege bleiben sie jedoch, was die Umsetzung angeht, auf der diskursiven Ebene der Überredung. Der Sprung in das geltende Recht gelingt nur über den Gesetzgeber, der die großen Zweckprogramme vorgibt. Nur er kann Sätze liefern, die hart genug sind, um auch gegen Widerstrebende durchgesetzt zu werden.

Demgegenüber wirken die publizistischen Anrufungen der "Solidarität " durchsetzungs- schwach. Sie erzeugen kaum mehr als "Stimmungen ", welche bei gegenläufigen wirtschaftlichen Interessen sofort wieder verfliegen. Gewiss sind Stimmungen nicht unwichtig, wie auch die ökonomische Theorie immer wieder betont. Bei karitativen Sammlungen spielt die »Solidarität mit den Opfern " eine erhebliche Rolle. Doch vergehen diese Stimmungen, wenn sie nicht in Steuer- oder Sozialrecht umgesetzt werden, bald wieder. Ihre Vergänglichkeit lässt sich nicht nur empirisch belegen, sondern auch soziologisch begründen. In einem Satz: »Alle überlieferten Formen bürgerlicher und brüderlicher Solidarität zersetzen sich in Folge funktionaler Differenzierung langsamer oder rascher ${ }^{\text {I }}{ }^{\mathbf{8}}$ Je mehr sich dieser Befund verfestigt und zu einer verlässlichen Aussage über das Innere moderner Gesellschaften wird, desto eher muss wohl das Misstrauen gegen einen zu großzügigen Gebrauch des Wortes »Solidarität « wachsen. Was heute im Inneren der westlichen Industriegesellschaften geschieht, kann weitaus besser mit Hilfe systemtheoretischer Modelle, mit der ökonomischen Analyse des Rechts und den Spiel- und Chaostheorien simuliert werden als mit den Gesellschaftstheorien des I9. Jahrhunderts, in denen "Solidarität « eine so große Rolle spielte. Dieses Wort bildet keine Realität mehr ab, und als Appellwort ist es verbraucht.

Michael Stolleis

\footnotetext{
I6 G. Frankenberg, Solidarität in einer "Gesellschaft der Individuen «?, in: Auf der Suche nach der gerechten Gesellschaft, hg. von DEMS., Frankfurt I994, 2 Io ff.; DERS., Die Verfassung der Republik. Autorität und Solidarität der Zivilgesellschaft, Baden-Baden I996, I $50 \mathrm{ff}$.

I7 Frankenberg, Solidarität (Fn. I6) $2 \mathrm{I} 4$.

ণัญ I8 Brunkhorst (Fn. 9) II 5 .
} 